\title{
Avaliação do Modelo de Monod para fermentação em caldo residual da casca de jabuticaba
}

\author{
Evaluation of the Monod Model for fermentation of the residual broth of jabuticaba bark \\ A. I. L. F. Nascimento ${ }^{1 *}$; D. G. Costa²; I. L. Dantas²; A. M. de Oliveira \\ Júnior'²; A. K. de S. Abud² \\ ${ }^{1}$ Departamento de Engenharia Química /Programa de Pós-Graduação em Engenharia Química, Universidade \\ Federal de Sergipe, CEP 49100-000, São Cristóvão-Sergipe, Brasil \\ ${ }^{2}$ Departamento de Engenharia de Alimentos, Universidade Federal de Sergipe, CEP 49100-000, São Cristóvão- \\ Sergipe, Brasil
}

*amanda_isabelly@yahoo.com.br

(Recebido em 25 de julho de 2017; aceito em 03 de julho de 2018)

\begin{abstract}
A jabuticaba Myrciaria cauliflora, fruta nativa brasileira, além de consumida em sua forma in natura, é utilizada para a produção de vários produtos, como geleias, sucos, bebidas alcoólicas fermentadas, entre outros. Há, no seu processamento, a geração significativa de resíduos, que muitas vezes são destinados para alimentação animal, adubo orgânico ou eliminados no meio ambiente sem tratamento. A busca de alternativas para aproveitamento dos resíduos produzidos, a fim de diminuir os impactos ambientais relacionados e agregar valor ao produto de origem é uma demanda significativa. Dentre os resíduos da jabuticaba pode-se citar a casca, que pode passar pelo processo de desidratação osmótica para obtenção de alguns subprodutos (jabuticaba passa e cristalizada). Esse processo gera um novo resíduo, que também pode ser reaproveitado, neste caso tem-se o caldo. O presente trabalho tem como objetivo apresentar uma descrição matemática da fermentação deste caldo que pode ser utilizado para a produção do vinho. $\mathrm{O}$ modelo matemático aqui proposto foi validado utilizando dados de um processo de fermentação real e será utilizado em trabalhos futuros de scale-up e controle do processo.
\end{abstract}

Palavras-chave: Casca de jabuticaba; cinética de fermentação; modelagem

Jaboticaba, Myrciaria cauliflora, a Brazilian native fruit, is largely eaten fresh, but it is also used for production of many products such as jams, juices, fermented beverages, among others. However, there is significant generation of waste, which often are intended for the production of animal feed, fertilizer or disposed of into the environment without treatment. Therefore, it is necessary to search for alternatives to use the waste produced by food industry, aiming to reduce environmental impacts and adding value to the product source. Peel is one of the residues from jaboticaba that can pass through the osmotic dehydration process to obtain some jaboticaba by-products (raisins or crystallized jaboticaba). This process generates another residue, which may also be reused: the syrup. The present project aims to describe mathematically the fermentation of this broth that is used for a production of vine. The mathematical model proposed here was validated using data from a real fermentation process and will be used in future scale-up and process control.

Keywords: Jaboticaba peel; fermentation kinetics, modeling

\section{INTRODUÇÃO}

O Brasil é o terceiro maior produtor mundial de frutas, atrás apenas da China e da Índia [1], tendo o Sudeste como principal polo de produção em âmbito nacional. A introdução da fruticultura nos vários estados cria oportunidades de emprego e de renda, bem como estimula a industrialização. Além dos frutos in natura oferecidos nos mercados regionais, a sua transformação em alimentos e bebidas possui larga aceitação, movimentando a economia das cidades.

Dentre as plantas frutíferas tropicais, a Jabuticabeira (Myrciaria jaboticaba) ocupa lugar de destaque devido à crescente comercialização de seus produtos principais: frutos, sucos e derivados 
como licores e análogos de vinho. É nativa da Mata Atlântica brasileira, presente principalmente na região Sudeste e parte do Sul [2].

Seus frutos, Jabuticabas, são arredondados, com casca de cor vermelha a preta, polpa esbranquiçada e doce, podendo conter de uma a quatro sementes. Possui alto valor nutricional, com alto teor de carboidratos, fibras, vitaminas, flavonóides e carotenóides e, ainda, sais minerais como ferro, cálcio e fósforo, principalmente em sua casca [3-4].

Apesar das características acima citadas, a jabuticaba não possui alto valor comercial, mesmo tendo uma larga produção em um único pé. Após a colheita, a fruta tem uma vida útil de dois a três dias, devido à rápida alteração da aparência, decorrente da intensa perda de água, deterioração e fermentação da polpa, o que prejudica a sua comercialização [5-6]. Potencial econômico há nesta fruta que pode ser consumida in natura ou utilizada pela indústria alimentícia como ingrediente, além de pesquisas relacionadas à sua utilização na indústria cosmética [7].

Em vista disso, o desenvolvimento de técnicas de preservação dos alimentos, mantendo o máximo dos componentes nutricionais e das propriedades organolépticas, é uma forma de viabilizar o aproveitamento racional. A desidratação osmótica tem se apresentado como uma ferramenta tecnológica importante nesse sentindo, permitindo o desenvolvimento de novos produtos derivados de frutas que, além de diminuir as perdas pós-colheita, aumenta o valor agregado da fruta sem perda do valor nutricional [8].

Este processo diminui a atividade da água na fruta e dificulta o crescimento e a reprodução da maioria das espécies de bactérias, leveduras e bolores [9-10]. Finalizada a desidratação, tem-se a calda como resíduo, que além de apresentar grande concentração de açúcares, ainda retém o aroma e o sabor da fruta. Esse resíduo pode então ser aproveitado para formulação de outros produtos [8].

A produção de vinho e destilado é um exemplo de reutilização do caldo, e este processo foi objeto de estudo, bem como sua modelagem.

Assim como outros processos de transformação, os processos fermentativos podem ser descritos quantitativamente, utilizando a modelagem matemática, que consiste, nesse caso, em escrever equações para os balanços de massa para cada componente do biorreator relacionados às complexas alterações bioquímicas que acontecem nos processos e às velocidades com que estas reações se processam [11].

O desenvolvimento de um modelo matemático para processos fermentativos possibilita atingir, dentre outros, os seguintes objetivos: estruturar de forma sucinta informações desconexas a respeito dos fenômenos biológicos num conjunto coerente; prever (e calcular) a respeito de quais elementos interações s são importantes num sistema complexo; encontrar novas técnicas para explicar o comportamento das células submetidas a determinados ambientes; reparar falhas eventualmente existentes no conhecimento convencional de determinados fenômenos e, por fim, entender as características qualitativamente essenciais de determinados processos [12].

O estudo da cinética de processos fermentativos não é algo recente. Monod, em 1949, associou a velocidade especifica de crescimento de microrganismos com a concentração de substrato [12]. O modelo por ele proposto é descrito classicamente pelo conjunto de equações (1) - (3) que seguem:

$$
\begin{gathered}
\frac{d X}{d t}=\mu_{x} X-k_{d} X \\
\frac{d P}{d t}=q X \\
\frac{d S}{d t}=\frac{1}{Y_{X / S}}\left(\frac{d X}{d t}\right)+\frac{1}{Y_{P / S}}\left(\frac{d P}{d t}\right)+m X
\end{gathered}
$$

Sendo $Y_{X / S}$ o fator de conversão substrato em células ( $\mathrm{kg}$ de células $/ \mathrm{kg}$ de substrato); $Y_{P / S} \mathrm{o}$ fator de conversão substrato em etanol ( $\mathrm{kg}$ de etanol $/ \mathrm{kg}$ de substrato); $\mu_{X}$ a velocidade específica máxima de crescimento celular $\left(\mathrm{s}^{-1}\right) ; P$ é a produtividade em etanol $\left(\mathrm{kg}\right.$ de etanol $\left./ \mathrm{m}^{3} . \mathrm{s}\right) ; X$ é a 
concentração de células no tempo $t\left(\mathrm{~kg} / \mathrm{m}^{3}\right) ; S$ a concentração de substrato no tempo $t\left(\mathrm{~kg} / \mathrm{m}^{3}\right)$ e $t$ $=$ tempo de fermentação (s).

Este trabalho apresenta o ajuste de parâmetros do processo de fermentação do caldo proveniente da desidratação osmótica da casca de jabuticaba (Myrciaria cauliflora) ao modelo cinético proposto por Monod, determinando os parâmetros envolvidos e validando o modelo a partir de dados de uma fermentação real.

\section{MATERIAL E MÉTODOS}

- Desidratação osmótica

As frutas foram adquiridas em comércio local e encaminhadas ao Departamento de Tecnologia de Alimentos da Universidade Federal de Sergipe, onde foram selecionadas e sanitizadas com solução de 200 ppm de cloro ativo por $10 \mathrm{~min}$ e, posteriormente, com solução de $3 \mathrm{ppm}$ de cloro ativo por $5 \mathrm{~min}$. As cascas foram separadas naturalmente da polpa e do caroço e, logo após, congeladas para futura utilização. Para a realização da desidratação osmótica, as cascas das jabuticabas foram descongeladas e imersas em uma solução de sacarose com $33^{\circ}$ Brix na proporção de 1:6 (casca: solução), a $40 \pm 2^{\circ} \mathrm{C}$ por duas horas. Após a desidratação osmótica, o caldo foi filtrado para remoção das cascas e, por fim, a matéria prima em questão foi obtida.

- Fermentação

O processo de fermentação foi executado em um biorreator de bancada de $5 \times 10^{-3} \mathrm{~m}^{3}$. O inóculo empregado para a fermentação foi a levedura Saccharomyces cerevisiae comercial, na forma de fermento biológico seco instantâneo, na concentração de $10 \mathrm{~kg} / \mathrm{m}^{3}$.

A fermentação foi realizada em reator de bancada em batelada, na temperatura de $30^{\circ} \mathrm{C}$, mantida através de sistema interno (camisa), com agitação de $50 \mathrm{rpm}$ e controle automático de $\mathrm{pH}$ na faixa de 3,7 a 4,3, sendo utilizadas soluções de carbonato de sódio $\left(\mathrm{Na}_{2} \mathrm{CO}_{3}\right) 15 \%(\mathrm{~m} / \mathrm{V})$ e/ou ácido cítrico $\left(\mathrm{C}_{6} \mathrm{H}_{8} \mathrm{O}_{7}\right) 5 \%(\mathrm{~m} / \mathrm{V})$. Durante o processo, amostras foram coletadas a fim de acompanhar a cinética da fermentação e possibilitar a determinação do final do processo através da leitura constante do teor de sólidos solúveis.

Após este processo, o fermentado foi resfriado para a temperatura de $4{ }^{\circ} \mathrm{C}$ para decantação da levedura, sofreu a primeira trasfega, e após 6 dias, foi feita a segunda trasfega, a fim de retirar os resíduos que se depositaram no fundo e clarificar a bebida, estando, então, pronto para ser utilizado na destilação.

Para o estudo da cinética da fermentação foi realizado o acompanhamento da concentração celular (biomassa), açúcares redutores totais (substrato) e etanol (produto) ao longo do tempo. Com base nos dados obtidos, foram calculados o rendimento em etanol ( $\mathrm{Y}_{\mathrm{P} / \mathrm{s}}, \mathrm{kg} / \mathrm{kg}$ ), a conversão de substrato em células $\left(\mathrm{Y}_{\mathrm{X} / \mathrm{s}}, \mathrm{kg} / \mathrm{kg}\right)$, a produtividade em etanol $\left(\mathrm{P}_{\mathrm{E}}, \mathrm{kg} / \mathrm{m}^{3} . \mathrm{s}\right)$ e a velocidade específica máxima de crescimento celular $\left(\mathrm{s}^{-1}\right)$, conforme expresso nas equações de (4) a (7)

$$
\begin{aligned}
& Y_{P / S}=\frac{P_{f}-P_{0}}{S_{0}-S_{f}} \\
& Y_{X / S}=\frac{X_{f}-X_{0}}{S_{0}-S_{f}} \\
& P_{E}=\frac{P_{f}-P_{0}}{t_{f}}
\end{aligned}
$$




$$
\mu_{X}=\frac{\ln X-\ln X_{0}}{t}
$$

Sendo $P_{E}$ é a produtividade em etanol (kg de etanol $\left./ \mathrm{m}^{3} . \mathrm{s}\right) ; X_{f}$ a concentração final de células $\left(\mathrm{kg} / \mathrm{m}^{3}\right) ; X_{0}$ é a concentração inicial de células $\left(\mathrm{kg} / \mathrm{m}^{3}\right) ; S_{f}$ a concentração final de substrato $\left(\mathrm{kg} / \mathrm{m}^{3}\right) ; S_{0}$ a concentração inicial de substrato $\left(\mathrm{kg} / \mathrm{m}^{3}\right) ; P_{f}$ é a concentração final de etanol $\left(\mathrm{kg} / \mathrm{m}^{3}\right) ; \mathrm{P}_{0}$ a concentração inicial de etanol $\left(\mathrm{kg} / \mathrm{m}^{3}\right) ; t_{f}$ o tempo total da fermentação $(\mathrm{s}) ;$

- Modelagem do processo fermentativo

O modelo cinético de Monod foi utilizado para descrever o comportamento dinâmico do processo. Funções e parâmetros foram implementados em código computacional no software Scilab ${ }^{\circledR}$ que forneceu a solução do modelo proposto para o processo.

Os parâmetros foram definidos através dos experimentos realizados e através de estudos efetuados durante esta etapa. E o modelo foi validado utilizando os dados da fermentação do caldo proveniente da desidratação osmótica da casca de jabuticaba.

\section{RESULTADOS E DISCUSSÃO}

Os dados utilizados para acompanhamento cinético da fermentação do caldo resultante da desidratação osmótica da casca de jabuticaba são apresentados na Figura 1.

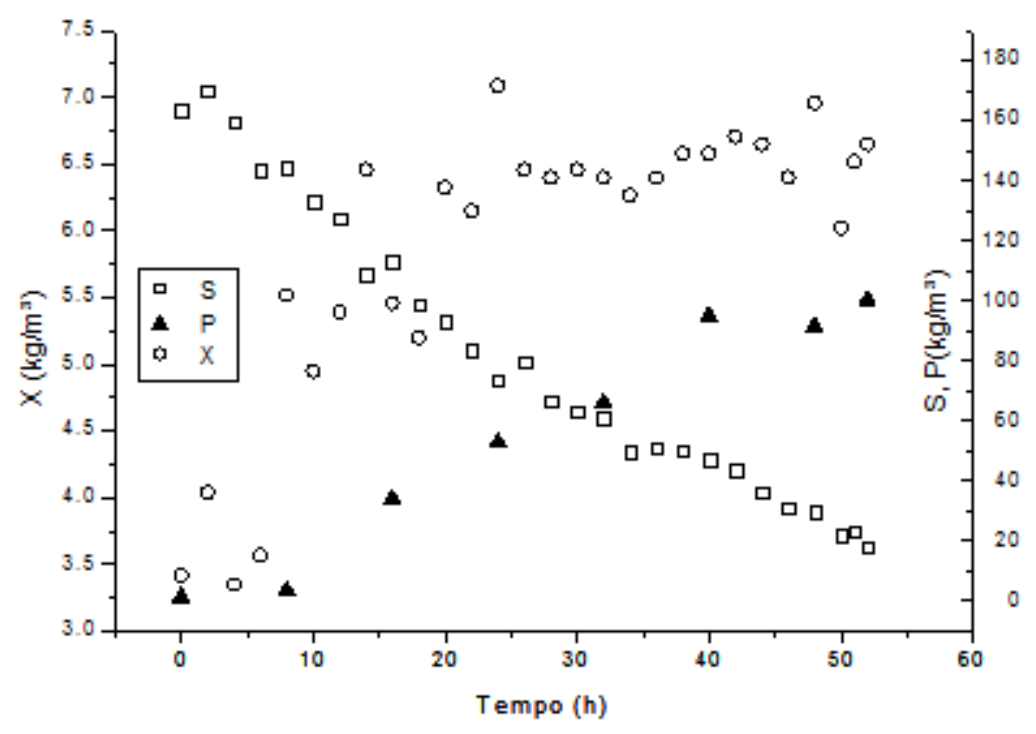

Figura 1: Comportamento cinético da fermentação

A variação de crescimento celular $(\Delta \mathrm{X})$, de consumo de substrato $(\Delta \mathrm{S})$ e de formação de etanol $(\Delta \mathrm{P})$ ao longo da fermentação foi obtida a partir dos dados acima apresentados. $\mathrm{E}$ ainda, a conversão de substrato em célula $\left(\mathrm{Y}_{\mathrm{X} / \mathrm{S}}\right)$, o rendimento em etanol $\left(\mathrm{Y}_{\mathrm{P} / \mathrm{S}}\right)$, a produtividade em etanol $\left(\mathrm{P}_{\mathrm{E}}\right)$ e a velocidade específica máxima de crescimento $\left(\mu_{\mathrm{x}}\right)$. Todos os valores obtidos seguem dispostos na Tabela 1. 
Tabela 1: Valores de parâmetros determinados dos dados da cinética de fermentação

\begin{tabular}{ccccccc}
\hline $\begin{array}{c}\Delta \mathrm{X} \\
\left(\mathrm{kg} / \mathrm{m}^{3}\right)\end{array}$ & $\begin{array}{c}\Delta \mathrm{S} \\
\left(\mathrm{kg} / \mathrm{m}^{3}\right)\end{array}$ & $\begin{array}{c}\Delta \mathrm{P} \\
\left(\mathrm{kg} / \mathrm{m}^{3}\right)\end{array}$ & $\begin{array}{c}\mathrm{Y}_{\mathrm{X} / \mathrm{s}} \\
(\mathrm{kg} / \mathrm{kg})\end{array}$ & $\begin{array}{c}\mathrm{Y}_{\mathrm{P} / \mathrm{S}} \\
(\mathrm{kg} / \mathrm{kg})\end{array}$ & $\mathrm{P}_{\mathrm{E}}\left(\mathrm{kg} / \mathrm{m}^{3} \cdot \mathrm{s}\right)$ & $\begin{array}{c}\mu_{x} \\
\left(\mathrm{~s}^{-1}\right)\end{array}$ \\
\hline 4,3 & 152,32 & 93,74 & 0,028 & 0,615 & $4,94 \times 10^{-4}$ & $1,86 \times 10^{-5}$ \\
\hline
\end{tabular}

A condição inicial do processo foi $\boldsymbol{X}_{\mathbf{0}}=3,5 \mathrm{~kg} / \mathrm{m}^{3}, \boldsymbol{S}_{\mathbf{0}}=160 \mathrm{~kg} / \mathrm{m}^{3}$ e $\boldsymbol{P}_{\mathbf{0}}=\mathbf{0}$. Os dados experimentais obtidos foram utilizados para validar um modelo matemático para o processo. Nesse sentido, utilizou-se o modelo clássico proposto por Monod para verificar se este descrevia de forma adequada o comportamento dinâmico do sistema.

Para tal, foram estabelecidos os parâmetros, inicialmente determinados a partir de dados da literatura e depois buscou-se valores que minimizavam o erro quadrático médio. A estratégia utilizada minimiza o erro gerado entre os dados experimentais e os dados resultantes do modelo. Os parâmetros estabelecidos para a verificação do modelo são apresentados na Tabela 2.

Tabela 2: Valores ajustados para o Modelo proposto por Monod para a Fermentação

\begin{tabular}{cc}
\hline Parâmetros & Valores ajustados modelo Monod \\
\hline $\boldsymbol{\mu}_{\mathbf{M A X}}$ & $1,16 \times 10^{-5}$ \\
$\mathbf{k}_{\mathbf{S}}$ & 80 \\
$\mathbf{Y}_{\mathbf{X S}}$ & 0,028 \\
$\mathbf{Y}_{\mathbf{P S}}$ & 0,615 \\
\hline
\end{tabular}

Os dados experimentais e os valores preditos pelo modelo foram confrontados a fim de verificar o ajuste do modelo. O comportamento obtido é apresentado na Figura 2 com os pontos discretos representando os dados experimentais e a linha contínua a predição do modelo.
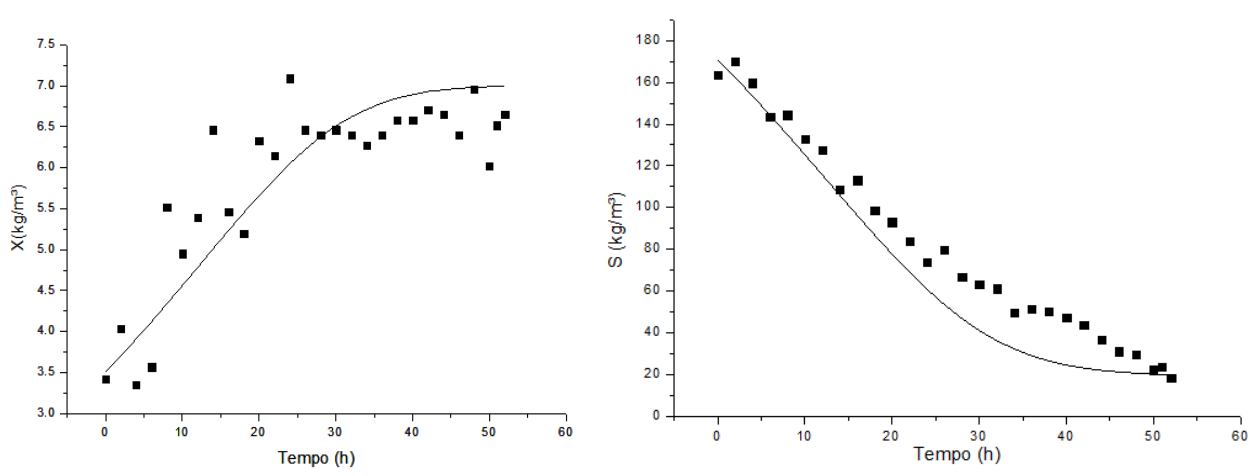

Figura 2: Validação do modelo proposto

Em relação aos dados experimentais, principalmente os observados na curva de crescimento de biomassa, há a presença de muitos "ruídos", podendo ser necessário um intervalo menor de tempo entre coletas de amostras, para melhor descrição do processo.

De forma geral, o comportamento do sistema é descrito de maneira satisfatória pelo modelo cinético de Monod utilizando os parâmetros listados anteriormente. Estudos fermentativos adicionais a fim de obter novos conjuntos de dados para o processo, e a busca por modelos matemáticos representativos, que não necessariamente o Monod, são requeridos. 


\section{CONCLUSÃO}

O modelo cinético de Monod apresentou uma descrição matemática adequada para descrever o comportamento da fermentação. $O$ estabelecimento de um modelo representativo para o processo é o primeiro passo para a realização de outros estudos como a busca por uma estratégia de controle adequada e o scale-up do processo.

\section{REFERÊNCIAS BIBLIOGRÁFICAS}

1. Anuário Bras. da Fruticultura 2015. Santa Cruz Do Sul: Editora Gazeta; 2015. 104 p.

2. Ascheri DPR, Andrade CT, Carvalho CWP, Ascheri JLR. Efeito da extrusão sobre a adsorção de água de farinhas mistas pré-gelatinizadas de arroz e bagaço de jabuticaba. Food Science and Tech. 2006 Jun;26(2):325-335, doi:S0101-20612006000200015

3. Garcia LGC. Aplicabilidade tecnológica da jabuticaba [dissertação]. Goiânia (GO): Universidade Federal de Goiás; 2014. 220 p.

4. Gomes AT, Cereda MP, Vilpoux O. Desidratação osmótica: uma tecnologia de baixo custo para o desenvolvimento da agricultura familiar. Rev Bras de Gestão e Desenv Reg. 2007;3(3):212-226.

5. Machado AMR, Santiago MCPA, Borguini RG, Godoy RLO, Gouvêa ACMS, Pacheco S, Nascimento LSM. Identificação e quantificação de antocianinas na casca de jabuticaba liofilizada. In: XVIII Encontro Nacional de Analistas de Alimentos; 2013; São Paulo: Soc. Bras. de Analistas de Alimentos; 2013. p. 904-907.

6. Daiuto ER, Vieites RL, Moraes MR, Evangelista RM. Conservação pós colheita de frutos de jabuticaba por irradiação. Rev Iberoamericana de Tecn Postcosecha. 2009;10(1):36-44, doi:813/81315095006

7. Alezandro MR, Granato D, Genovese MI. Jaboticaba (Myrciaria jaboticaba (Vell.) Berg), a Brazilian grape-like fruit, improves plasma lipid profile in streptozotocin-mediated oxidative stress in diabetic rats. Food Res International. 2013 Nov;54(1):650-659, doi:S0963996913004079

8. Oliveira LA, Lordelo F. dos S., Tavares JTQ, Cazetta ML. Elaboração de bebida fermentada utilizando calda residual da desidratação osmótica de abacaxi (Ananas comosus l). Rev Bras de Tecn Agroindustrial. 2012;6(1):702-712, doi:10.3895/S1981-36862012000100009

9. Gava AJ, Silva CAB, Frias JRG. Tecn. de Alimentos: Princípios e Aplicações. São Paulo: Nobel; 2009. p. 364.

10. Gomes AT, Cereda MP, Vilpoux O. Desidratação Osmótica: uma tecnologia de baixo custo para o desenvolvimento da agricultura familiar. Rev Bras de Gestão e Desenv Regional. 2007;3(3):212-226.

11. Ferreira RM. Modelagem e simulação de biorreatores tipo torre operando com leveduras autoimobilizáveis para produção de etanol. [Tese]. Campinas (SP): Universidade Estadual de Campinas, Campinas - SP; 2013. 154p.

12. Borzani W, Aquarone E, Lima UA, Schimidell W. Biotecnologia Industrial. São Paulo: Edgard Blucher; 2001. p. 522. 